\title{
Coexistence of Active Species in Anionic Polymerization of Ethyl Methacrylate by Butyllithium
}

\author{
Koichi Hatada, Tatsuki Kitayama, Hikaru Sugino \\ Yoshihiro Umemura, Mitsuru Furomoto, and Heimei YuKI \\ Department of Chemistry, Faculty of Engineering Science, Osaka University, \\ Toyonaka, Osaka 560, Japan.
}

(Received August 13, 1979)

\begin{abstract}
Polymerizations of ethyl methacrylate were carried out in toluene with butyllithium at various temperatures. The polymer obtained at $-78^{\circ} \mathrm{C}$ could be fractionated into methanol-insoluble and methanol-soluble fractions. The former was rather syndiotactic. The latter was found by gel-permeation chromatography and ${ }^{1} \mathrm{H}$ NMR spectroscopy to be composed of highly isotactic polymer and isotactic oligomer. These three fractions were formed simultaneously at the beginning of the polymerization and the amounts and molecular weights of the isotactic and syndiotactic fractions increased during the polymerization, while the amount of oligomeric fraction remained almost constant after the initial rapid increase. These results indicate the coexistence of three different active species for isotactic and syndiotactic polymers and isotactic oligomer, respectively, at the initial stage of the polymerization and two propagating species during the polymerization. The amounts of syndiotactic fraction and oligomeric fraction decreased with an increase in the polymerization temperature and only the isotactic polymer was obtained above $-20^{\circ} \mathrm{C}$. When the polymerization was initiated with 1,1-diphenylhexyllithium, only an isotactic polymer was produced. In the polymerization initiated with butyllithium, an appreciable amount of lithium ethoxide formed at the initial stage of the reaction, but no formation of the ethoxide was observed in the case of 1,1-diphenylhexyllithium. This indicates that the multiplicity of the active species in the polymerization with butyllithium is strongly related to the lithium ethoxide formed.

KEY WORDS Anionic Polymerization / Poly(ethyl methacrylate) / Poly(tert-butyl methacrylate) / Butyllithium / 1,1-Diphenylhexyllithium / Lithium Ethoxide / Stereoregulated Polymerization / Coexistence of Propagating Species /
\end{abstract}

It has been suggested that the broad molecular weight distribution of poly(methyl methacrylate) prepared in toluene with anionic initiator can be explained in terms of a combination of two narrower distributions produced by simultaneously occcurring mechanisms. ${ }^{1}$ Cottam et $a l^{2}{ }^{2}$ suggested the existence of two active species uncomplexed and complexed with lithium methoxide, respectively, in the polymerization of methyl methacrylate with butyllithium (BuLi). Recently, it was reported that in the polymerization of methyl methacrylate in a mixture of toluene and tetrahydrofuran with Grignard reagent, the polymers obtained consist of two components, methanol-soluble and methanolinsoluble polymers, which are considered to form at two distinct and independent active centers. ${ }^{3}$
In a previous paper, ${ }^{4}$ we postulated the existence of two types of active species in the polymerization of methyl $\alpha$-ethylacrylate by $\mathrm{BuLi}$ in toluene, which produce isotactic and syndiotactic polymers, respectively. In the present work, it is suggested that three kinds of active species exist in the anionic polymerization of ethyl methacrylate (EMA) initiated by BuLi in toluene.

\section{EXPERIMENTAL}

Ethyl and tert-butyl methacrylates were obtained from a commercial source and purified by fractional distillation under nitrogen pressure. The monomers thus purified were distilled over calcium hydride under high vacuum. 
BuLi was prepared from butyl chloride and metallic lithium in heptane under dry argon. 1,1Diphenylhexyllithium was synthesized by the reaction of equimolar amounts of $\mathrm{BuLi}$ and 1,1diphenylethylene in toluene.

Polymerization was carried out in a glass ampoule under dry nitrogen. The reaction was stopped by adding a small amount of methanol, and the mixture was poured into a large amount of methanol. After standing overnight, the precipitated polymer was collected by filtration, washed with methanol and dried in vacuo at room temperature. The combined filtrate was evaporated under reduced nitrogen pressure. The residue was dissolved in benzene and a small amount of insoluble material was removed by filtration. The fraction soluble in methanol was recovered by freeze-drying from benzene.

In the polymerization designed to detect the lithium alkoxide the reaction was stopped by adding a small amount of acetic acid, and the mixture was analyzed for the alcohol by gas-liquid chromatography. It was assumed that the alkoxide formed during the polymerization reaction was converted quantitatively to the corresponding alcohol. ${ }^{5}$

The ${ }^{1} \mathrm{H}$ NMR spectra were taken on a JNM-FX100 (JEOL) spectrometer at $100 \mathrm{MHz}$. The triad tacticities of poly(ethyl methacrylate)s were measured by a peak eliminated Fourier transform NMR method $^{6}$ in toluene- $d_{8}$ at $100^{\circ} \mathrm{C}$
Gel-permeation chromatography (GPC) was performed with a JASCO model FLC-A 10 with Shodex GPC column A-80M with maximum porosity of $5 \times 10^{7}$ using tetrahydrofuran as a solvent. The chromatogram was calibrated against standard polystyrene samples. The yield and molecular weight of isotactic polymer and oligomer were estimated by splitting the bimodal chromatogram of the methanol-soluble fraction into two components.

\section{RESULTS}

The polymerizations of EMA were carried out in toluene with BuLi as an initiator at various temperatures for $24 \mathrm{~h}$. The results are shown in Table I. The polymer obtained at $-78^{\circ} \mathrm{C}$ could be fractionated into a methanol-soluble and a methanol-insoluble fraction, which were proved to be isotactic and rather syndiotactic, respectively, by NMR spectroscopy. The gel-permeation chromatogram of the isotactic fraction showed the high- and lowmolecular-weight peaks as illustrated in Figure 1. The methanol-soluble fraction prepared at $-78^{\circ} \mathrm{C}$ was separated into two portions at the elution volume of $40.6 \mathrm{ml}$. The isotacticity of the high molecular weight part $(I 89, H 6, S 5 \%)$ was found to be higher than that of low-molecular-weight one ( $I$ $74, H 14, S 12 \%$ ). The methanol-insoluble fraction showed a single peak in the gel-permeation chroma-

Table I. Polymerization of EMA in toluene by BuLi at various temperatures for $24 \mathrm{~h}^{\mathrm{a}}$

\begin{tabular}{|c|c|c|c|c|c|c|c|c|c|c|c|}
\hline \multirow{3}{*}{ No. } & \multirow{3}{*}{ Temp $/{ }^{\circ} \mathrm{C}$} & \multicolumn{4}{|c|}{ Insoluble in $\mathrm{MeOH}$} & \multicolumn{6}{|c|}{ Soluble in $\mathrm{MeOH}$} \\
\hline & & \multirow{2}{*}{ Yield $/ \%$} & \multicolumn{3}{|c|}{ Tacticity $/ \%$} & \multirow{2}{*}{ Yield $/ \%$} & \multicolumn{3}{|c|}{ Tacticity $/ \%$} & \multirow{2}{*}{ Isotactic $/ \%$} & \multirow{2}{*}{ Oligomeric $\mathrm{d} / \%$} \\
\hline & & & $I$ & $H$ & $S$ & & $I$ & $H$ & $S$ & & \\
\hline 1 & -78 & 18 & 19 & 33 & 48 & 81 & 80 & 14 & 6 & 52 & 29 \\
\hline 2 & -40 & 6 & 44 & 25 & 31 & 88 & 79 & 14 & 7 & 70 & 18 \\
\hline 3 & -20 & 0 & - & - & - & 94 & 81 & 13 & 6 & 94 & 0 \\
\hline 4 & 0 & 0 & - & - & - & 94 & 78 & 17 & 5 & 94 & 0 \\
\hline 5 & $-78^{b}$ & 2 & 75 & 14 & 11 & 98 & 77 & 16 & 7 & 100 & 0 \\
\hline 6 & $-78^{\mathrm{c}}$ & 1 & 9 & 32 & 59 & 99 & 79 & 16 & 5 & $-^{e}$ & $-^{\mathrm{e}}$ \\
\hline
\end{tabular}

a EMA, $10 \mathrm{mmol}$; toluene, $10 \mathrm{ml}$; BuLi, $0.3 \mathrm{mmol}$.

b Initiated with $0.3 \mathrm{mmol}$ of 1,1-diphenylhexyllithium.

c Initiated with $0.3 \mathrm{mmol}$ of 1,1-diphenylhexyllithium in the presence of $0.2 \mathrm{mmol}$ of lithium ethoxide.

d The amounts of isotactic polymer and oligomer were estimated from the bimodal gel permeation chromatograms of the methanol-soluble fraction.

e It was not possible to detect the existence of oligomeric fraction from the GPC chromatogram. 


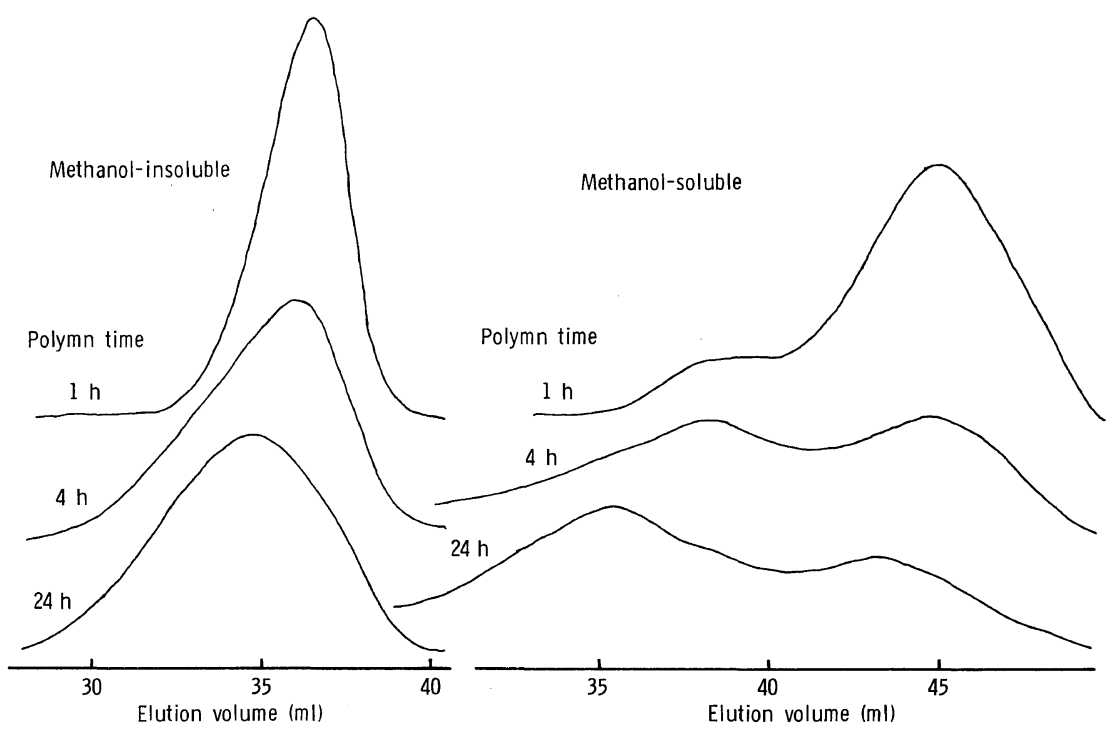

Figure 1. Gel permeation chromatograms of poly(ethyl methacrylate)s prepared in toluene with BuLi at $-78^{\circ} \mathrm{C}$ for various polymerization times.

togram. So the polymer obtained at $-78^{\circ} \mathrm{C}$ consists of isotactic and syndiotactic polymers and isotactic oligomers.

The time dependence of the polymerization of EMA in toluene with $\mathrm{BuLi}$ at $-78^{\circ} \mathrm{C}$ is given in Table II. The gel-permeation chromatograms of the methanol-soluble and methanol-insoluble fractions are shown in Figure 1. The yield and the molecular weight of isotactic polymer and oligomer estimated from the bimodal chromatogram of the methanolsoluble fraction are shown in Table II. With an increase in the polymerization time, the yield and the molecular weight of isotactic and syndiotactic polymers increased and the molecular weight distribution for both fractions became gradually wide. The oligomeric product appears to form rapidly at the early stage of the polymerization and the $M_{w} / M_{n}$ ratio stays relatively constant in the course of the polymerization. The amount in millimole of polymer molecules formed was calculated and is listed in Table III. The amounts in millimoles for isotactic and syndiotactic polymers gradually increased during the polymerization, while the amount of the oligomer molecules remained almost constant.

By increasing the polymerization temperature, the amount of the methanol-insoluble fraction decreased and only a soluble and isotactic fraction was obtained above $-20^{\circ} \mathrm{C}$. The insoluble fraction obtained at $-40^{\circ} \mathrm{C}$ was not syndiotactic but rather stereoblock. The gel permeation chromatograms of the methanol-soluble fractions revealed that the oligomeric product also decreased in its amount with

Table II. Polymerization of EMA in toluene with BuLi at $-78^{\circ} \mathrm{C}$ for various polymerization times ${ }^{\mathrm{a}}$

\begin{tabular}{|c|c|c|c|c|c|c|c|c|c|}
\hline \multirow{2}{*}{ Time/h } & \multicolumn{3}{|c|}{ Syndiotactic } & \multicolumn{3}{|c|}{ Isotactic } & \multicolumn{3}{|c|}{ Oligomeric } \\
\hline & Yield $/ \%$ & $M_{n} \times 10^{-3}$ & $M_{w} / M_{n}$ & Yield $/ \%$ & $M_{n} \times 10^{-3}$ & $M_{w} / M_{n}$ & Yield $/ \%$ & $M_{n} \times 10^{-3}$ & $M_{w} / M_{n}$ \\
\hline 1 & 1.8 & 143.2 & 1.23 & 3.6 & 49.4 & 1.34 & 20.5 & 2.57 & 2.29 \\
\hline 4 & 8.5 & 179.2 & 1.66 & 24.8 & 63.4 & 2.33 & 24.8 & 3.34 & 2.24 \\
\hline 24 & 18.0 & 201.5 & 1.86 & 51.8 & 113.0 & 2.38 & 29.2 & 4.13 & 2.79 \\
\hline
\end{tabular}

${ }^{\text {a }}$ EMA, $10 \mathrm{mmol}$; toluene, $10 \mathrm{ml}$; BuLi, $0.3 \mathrm{mmol} . M_{n}$ and $M_{w} / M_{n}$ were determined from the GPC chromatogram calibrated against standard polystyrene samples. 
Table III. Amount of polymer molecules formed during the polymerization in toluene with $\mathrm{BuLi}$ at $-78^{\circ} \mathrm{C}^{\mathrm{a}}$

\begin{tabular}{|c|c|c|c|c|c|c|}
\hline \multirow{2}{*}{$\begin{array}{l}\text { Polymerization } \\
\text { time }\end{array}$} & \multicolumn{2}{|c|}{ Syndiotactic } & \multicolumn{2}{|c|}{ Isotactic } & \multicolumn{2}{|c|}{ Oligomeric } \\
\hline & \multicolumn{2}{|c|}{ Yield } & \multicolumn{2}{|c|}{ Yield } & \multicolumn{2}{|c|}{ Yield } \\
\hline $\mathrm{h}$ & $\%$ & mmol & $\%$ & mmol & $\%$ & mmol \\
\hline 1 & 1.8 & 0.0001 & 3.6 & 0.0008 & 20.5 & 0.091 \\
\hline 4 & 8.5 & 0.0005 & 24.8 & 0.0045 & 24.8 & 0.085 \\
\hline 24 & 18.0 & 0.0010 & 51.8 & 0.0052 & 29.2 & 0.081 \\
\hline
\end{tabular}

${ }^{\text {a }}$ EMA, $10 \mathrm{mmol}$; toluene, $10 \mathrm{ml}$; BuLi, $0.3 \mathrm{mmol}$.

Table IV. Polymerization of EMA in toluene with $\mathrm{BuLi}$ at $-20^{\circ} \mathrm{C}$ for various polymerization times ${ }^{\mathrm{a}}$

\begin{tabular}{|c|c|c|c|c|c|c|c|}
\hline \multirow{3}{*}{ Time $/ \mathrm{s}$} & \multicolumn{7}{|c|}{ Soluble in $\mathrm{MeOH}$} \\
\hline & \multirow{2}{*}{ Yield $/ \%$} & \multicolumn{3}{|c|}{ Tacticity $/ \%$} & \multirow{2}{*}{$M_{n} \times 10^{-3}$} & \multirow{2}{*}{$M_{w} / M_{n}$} & \multirow{2}{*}{$N^{\mathrm{b}}$} \\
\hline & & $I$ & $H$ & $S$ & & & \\
\hline 30 & 39 & 73 & 18 & 9 & 10.29 & 3.04 & 0.043 \\
\hline 90 & 90 & 76 & 18 & 6 & 25.98 & 1.84 & 0.039 \\
\hline
\end{tabular}

${ }^{a}$ EMA, $10 \mathrm{mmol}$; toluene, $10 \mathrm{ml}$; BuLi, $0.3 \mathrm{mmol} . M_{n}$ and $M_{w} / M_{n}$ were determined from the GPC chromatogram calibrated against standard polystyrene samples.

b Amount in millimole of polymer molecules formed during the polymerization.

an increase in the polymerization temperature and was not formed above $-20^{\circ} \mathrm{C}$ (Table I).

The effect of the time on the polymerization at $-20^{\circ} \mathrm{C}$ is shown in Table IV. The reaction was very rapid at this temperature and almost finished in 90 seconds. The polymer obtained was methanolsoluble and isotactic, and showed a single peak in the GPC chromatogram, indicating the absence of the oligomeric fraction. The polymer formed for the first 30 seconds was low in the molecular weight and broad in the molecular weight distribution. With increasing the polymerization time the molecular weight increased and the distribution became narrower.

\section{DISCUSSION}

As was mentioned above the poly(ethyl methacrylate) obtained by $\mathrm{BuLi}$ at $-78^{\circ} \mathrm{C}$ in toluene ,consists of the syndiotactic, isotactic, and oligomeric fractions. The results could be explained by the coexistence of three different active species. The experiments for the different polymerization times revealed that these three fractions appeared simultaneously at the initial stage of the reaction, and the isotactic and syndiotactic fractions increased in the amount and the molecular weight with an increase in the polymerization time, while the amount of oligomeric fraction remained almost constant after the initial rapid increase (Figure 1 and Table II). This indicates that there exist three different active species at the initial stage of the polymerization and two propagating species through the polymerization.

In the polymerization of methyl methacrylate (MMA) with BuLi, the initiator disappears almost instantaneously on mixing the reactants ${ }^{2.7}$ and reacts first with the carbonyl groups to produce lithium methoxide than with the monomer vinyl bonds at a lower temperature. ${ }^{8}$ When 1,1-diphenylhexyllithium was used as the initiator, the amount of lithium methoxide formed was much less than with $\mathrm{BuLi}$, and the isotacticity was lower for the butyllithium-initiated polymer than for diphenylhexyllithium-initiated one. ${ }^{8}$ From these results, it is suggested that lithium methoxide in the reaction mixture was related to the formation of syndiotactic 
blocks in the polymer. ${ }^{8}$

When the polymerization of EMA was carried out in toluene with 1,1-diphenylhexyllithium as an initiator at $-78^{\circ} \mathrm{C}$, a large amount of methanolsoluble, isotactic polymer was formed with a very small amount of the methanol-insoluble fraction. The latter was also isotactic in this case (No. 5 in Table I). The soluble fraction showed a single peak in the gel-permeation chromatogram.

Lithium ethoxide formed at the initial stage of the reaction was analyzed for the polymerizations of EMA by 1,1-diphenylhexyllithium and $\mathrm{BuLi}$ at $-78^{\circ} \mathrm{C}$ in toluene (Table V). No formation of lithium ethoxide was observed for the polymerization by 1,1-diphenylhexyllithium while an appreciable amount of the ethoxide formed in the polymerization by BuLi. When the polymerization initiated with 1,1-diphenylhexyllithium was carried out in the presence of the added lithium ethoxide, a small amount of syndiotactic polymer formed with a large amount of isotactic polymer (No. 6 in Table I), indicating an important role of the ethoxide for the formation of syndiotactic polymer. The amount of the syndiotactic polymer formed in the polymerization by 1,1-diphenylhexyllithium in the presence of the added ethoxide was smaller than that of syndiotactic polymer formed in the polymerization by BuLi. The situation may be somewhat different if the preformed ethoxide is added instead of ethoxide formed in situ. The formation of the alkoxide greatly decreased in the polymerization of $t$-butyl methacrylate (Table V), which gave only highly isotactic polymer in toluene by $\mathrm{BuLi}$.

Table V. Lithium alkoxide formed for the first 10 minutes in the polymerization of alkyl methacrylate in toluene at $-78^{\circ} \mathrm{C}$

\begin{tabular}{|c|c|c|c|}
\hline \multirow{2}{*}{ Alkyl } & \multirow{2}{*}{ Initiator } & \multicolumn{2}{|c|}{ LiOR formed } \\
\hline & & mmol & $\% \mathrm{~b}$ \\
\hline $\mathrm{C}_{2} \mathrm{H}_{5}$ & $\mathrm{C}_{4} \mathrm{H}_{9} \mathrm{Li}$ & 0.12 & 25 \\
\hline $\mathrm{C}_{2} \mathrm{H}_{5}$ & $\mathrm{DPHLi}^{\mathrm{c}}$ & 0.00 & 0 \\
\hline$t-\mathrm{C}_{4} \mathrm{H}_{9}$ & $\mathrm{C}_{4} \mathrm{H}_{9} \mathrm{Li}$ & 0.04 & 8 \\
\hline
\end{tabular}

a Monomer, $10 \mathrm{mmol}$; initiator, $0.5 \mathrm{mmol}$; solvent, $10 \mathrm{ml}$; polymerization time, $10 \mathrm{~min}$. The polymerization was terminated by the addition of $1.0 \mathrm{mmol}$ of acetic aid.

b Percentage based on the initiator used.

c 1,1-Diphenylhexyllithium.
The facts mentioned here indicate that the multiplicity of the active species in the polymerization of EMA with BuLi is closely related to the lithium ethoxide formed at the initial stage of the polymerization. The active species for the syndiotactic fraction may exist in the form of ion pairs complexed with one or more molecules of lithium ethoxide, while the isotactic active species are associated less with the ethoxide or free from it. At higher polymerization temperatures, the coordination of ethoxide weakens, resulting in a decrease in the formation of the syndiotactic, methanol-insoluble fraction, and only the isotactic polymer is formed at $-20^{\circ} \mathrm{C}$. The methanol-insoluble fraction prepared at $-40^{\circ} \mathrm{C}$ had a rather stereoblock structure. This may be due to the existence of more and less complexed propagating species with lithium ethoxide, which slowly interconvert. Formation of the oligomer also seems related to the lithium ethoxide since the variation in the amount of oligomer in considerations of the reaction conditions is similar to that of syndiotactic polymer, but the true mechanism is not clear at present.

As shown in Table III the amounts in millimoles of isotactic and syndiotactic polymer molecules increased in the course of the polymerization at $-78^{\circ} \mathrm{C}$. In the polymerization of methyl methacrylate in toluene with alkyllithium, no termination reaction occurred at the growing chain ends for a high molecular weight polymer ${ }^{8.9}$ and the initiator disappeared instantaneously on mixing the reactants. ${ }^{2.7}$ On the other hand, the oligomeric chain ends were essentially inactive at the later stage of the polymerization but most of them were not really terminated and still reactive to the proton of methanol. ${ }^{9,10}$ So, the increase in the number of polymer molecules mentioned above should be due to the transformation of a part of the dormant oligomeric chains into the propagating species for isotactic or syndiotactic polymer during the polymerization. It is not clear at present how the oligomer chain terminates its further growth at the early stage of the polymerization and how the dormant species of the oligomer are sometimes awaken and come to take on the monomer rapidly as the propagating species of the isotactic or syndiotactic polymer. Further study should be made on this point.

Acknowledgements. The authors are very grateful to Professor H. Höcker of Bayreuth University, 


\section{K. Hatada et al.}

Dr. A. H. E. Müller of University of Mainz, and Professor M. Van Beylen of University of Leuven for their helpful comments. Thanks are also due to Mrs. F. Yano for her clerical assistance in preparing this manuscript.

\section{REFERENCES}

1. T. J. R. Weakley, R. J. P. Williams, and J. D. Wilson, J. Chem. Soc., 3963 (1960).

2. B. J. Cottam, D. M. Wiles, and S. Bywater, Can. J. Chem., 41, 1905 (1963).

3. B. O. Bateup and P. E. M. Allen, Eur. Polym. J., 13, 761 (1977)
4. K. Hatada, S. Kokan, T. Niinomi, K. Miyaji, and H. Yuki, J. Polym. Sci., Polym. Chem. Ed., 13, 2117 (1975).

5. D. M. Wiles and S. Bywater, J. Phys. Chem., 68, 1983 (1964).

6. K. Hatada, K. Ohta, Y. Okamoto, T. Kitayama, Y. Umemura, and H. Yuki, J. Polym. Sci., Polym. Lett. Ed., 14, 531 (1976).

7. D. L. Glusker, E. Stiles, and B. Yoncoskie, J. Polym. Sci., 49, 297 (1961).

8. D. M. Wiles and S. Bywater, Trans. Faraday Soc., 61, 150 (1965).

9. K. Hatada, T. Kitayama, K. Fujikawa, K. Ohta, and H. Yuki, Polym. Bull., 1, 103 (1978).

10. D. L. Glusker, I. Lysloff, and E. Stiles, J. Polym. Sci., 49, 315 (1961). 